\title{
Electrical Conductivity of Films Formed by Few-Layer Graphene Structures Obtained by Plasma-Assisted Electrochemical Exfoliation of Graphite
}

\author{
Vladimir P. Vasiliev $(\mathbb{D}$, Roman A. Manzhos $(\mathbb{D}$, and Alexander G. Krivenko \\ Institute of Problems of Chemical Physics of Russian Academy of Sciences, Academician Semenov avenue 1, Chernogolovka, \\ Moscow 142432, Russia
}

Correspondence should be addressed to Vladimir P. Vasiliev; vpvasiliev@mail.ru

Received 30 November 2018; Revised 12 February 2019; Accepted 11 March 2019; Published 28 April 2019

Academic Editor: Gerd-Uwe Flechsig

Copyright (C) 2019 Vladimir P. Vasiliev et al. This is an open access article distributed under the Creative Commons Attribution License, which permits unrestricted use, distribution, and reproduction in any medium, provided the original work is properly cited.

Current-voltage characteristics of few-layer graphene structures (FLGS) obtained by plasma-assisted electrochemical exfoliation of graphite in $\mathrm{Na}_{2} \mathrm{SO}_{4}$ solution were measured. FLGS are shown to possess electronic conductivity, which indicates the predominant functionalization of the edges of graphene planes and the preservation of the structure of basal planes in obtained nanostructures as in the source graphite. The effect of humidity on the conductivity of FLGS films was studied. The resistance of films was found to increase with an increase in the relative humidity of the environment due to the shielding of FLGS flakes by a film of water. The effect of different solvents on the current-voltage characteristics of FLGS was analyzed. The conductivity of films significantly decreased in vapors of polar protic solvents, while there was a minor effect of nonpolar aprotic solvents on the conductivity of FLGS films.

\section{Introduction}

Mass production of high-quality graphene is important for its application in next-generation electronics $[1,2]$, composite materials [3, 4], energy storage devices [5], and development of platinum metals free catalysts for oxygen reduction reaction (ORR) [6-10]. High activity in ORR was demonstrated for carbon nanostructures doped with the atoms of $p$ elements.

A prerequisite for the widespread use of materials based on graphene is a simple and reliable way of its production. At present, among the diverse methods of synthesis of few-layer graphene structures (FLGS), the method of electrochemical exfoliation of graphite rapidly gains its popularity [11]. This is due to a number of advantages of this approach compared to other conventional methods of FLGS production: the simplicity of purification of synthesized material, the ability to control degree of oxidation and defectiveness of graphene particles, one-step and environmental friendly method (the approach utilizes solutions of salts with a moderate concentration, unlike all chemical methods using concentrated acids).

Graphite is believed to be chemically inert, but various functional groups, e.g., $-\mathrm{COOH},-\mathrm{OH},=\mathrm{O},-\mathrm{O}-$, and other defects are formed on carbon atoms located on the basal planes and edges in the course of FLGS synthesis. The interaction of functional groups on FLGS surface affects the stacking of layers in flakes of few-layer graphene structures.

The conductivity of graphene and various graphene structures is the subject of thorough attention associated, primarily, with the potential use of these materials in nanoelectronic devices [12]. Electrical conductivity of graphene with an ideal structure was believed to exceed the corresponding value for real graphene with structural defects; however, this point of view was disproved by experimental and theoretical studies that have shown an increase in the electrical conductivity of graphene as a result of an increase in the number of defects in graphene sheet (see, for example, [13]). At the same time, 
TABLE 1: Cls peak data for c-FLGS and a-FLGS.

\begin{tabular}{lcccc}
\hline Sample & C-C (at.\%) & C-OH (at.\%) & C-O-C (at.\%) & O-C=O (at.\%) \\
\hline c-FLGS & 59.2 & 7.7 & 30.2 & 2.8 \\
a-FLGS & 54.6 & 8.8 & 23.9 & 12.8 \\
\hline
\end{tabular}

FLGS used as catalysts for ORR should possess sufficient electronic conductivity. For example, as nitrogen content increases in $\mathrm{N}$-doped graphene, its conductivity decreases, which leads to a decrease in the catalytic activity of these materials towards oxygen reduction reaction [8].

Thus, determination of conductivity of films formed by graphene structures is of both fundamental and applied importance. In this study, we propose a simple and effective method to estimate the resistance of films formed by few-layer graphene structures obtained by plasma-assisted electrochemical exfoliation of graphite electrodes in $\mathrm{Na}_{2} \mathrm{SO}_{4}$ solution. The analysis of influence of humidity and solvent nature on the conductivity of FLGS films was carried out.

\section{Experimental Section}

FLGS were synthesized by the method of plasma-assisted electrochemical exfoliation of graphite using high voltage pulses [14]. The process was carried out with the homemade set-up [15], which allows one to apply to graphite electrodes immersed in an electrolyte solution a sequence of voltage pulses with an amplitude of up to $250 \mathrm{~V}$ and a rise time of $c a 0.5 \mu \mathrm{s}$. A high current density $\left(>20 \mathrm{~A} / \mathrm{cm}^{2}\right)$ on the electrodes is a necessary condition for the formation of electrolytic plasma [14]. For graphite electrodes significantly different in size, intense acoustic and light generation was observed around the electrode with a smaller size. Thus, depending on the polarity of voltage pulses, either cathodic or anodic electrolytic plasma was generated [14]. FLGS particles obtained using cathodic (c-FLGS) or anodic plasma (a-FLGS) were separated from electrolyte and washed with distilled water during several cycles of centrifugation.

Current-voltage characteristics of films were obtained by two-point method using an Elins P-20X potentiostat (Elins, Russia). The measurements were carried out on $9 \mathrm{~mm} \times 20$ $\mathrm{mm}$ contact plate in an environment with certain content of water vapor [16] or another solvent. Contact plate was an interdigitated electrode with a $0.3 \mathrm{~mm}$ gap between $4.5 \mathrm{~mm} \times$ $0.2 \mathrm{~mm}$ contacts. Noteworthy, the distance between contacts substantially exceeds lateral size of separate FLGS flakes. 250 $\mu \mathrm{L}$ of FLGS aqueous suspension with concentration of 0.4 $\mathrm{mg} / \mathrm{mL}$ was drop-casted onto contact plate; and the film was dried in air at room temperature during several hours. The electrical conductivity of FLGS films was estimated from the current observed at a constant voltage of $300 \mathrm{mV}$. Sample was first placed in a tube with water (or another solvent) and then in a tube with a drying agent (calcium chloride). The plate itself was not in direct contact with a liquid and a drying agent, but was in solvent vapors.

The size distribution of FLGS particles was obtained using an LS 13320 XR laser particle size analyzer (Beckman Coulter, USA). The thickness of FLGS films (200-300 nm) was determined by the concentration of the initial FLGS suspension $(0.4 \mathrm{mg} / \mathrm{mL})$ and monitored using an SOLVER Open (NT-MDT Spectrum Instruments, Russia) atomic force microscope (AFM). The X-ray photoelectron spectra (XPS) were obtained using an SPECS PHOIBOS 150 MCD electron spectrometer (SPECS, Germany) and X-ray tube with a magnesium anode $(\mathrm{h} v=1253.6 \mathrm{eV})$. The vacuum in the spectrometer chamber did not exceed $4 \times 10^{-8} \mathrm{~Pa}$. The spectra were registered in the regime of constant transmission energy ( $40 \mathrm{eV}$ for the wide spectra and $10 \mathrm{eV}$ for individual lines). The survey spectrum was recorded in steps of $1.00 \mathrm{eV}$, the spectra of individual lines were recorded in steps of $0.03 \mathrm{eV}$.

\section{Results and Discussion}

Graphene-like structures obtained in the present study are particles with a wide distribution of lateral size $d$, which varies from 60 to $550 \mathrm{~nm}$ (Figure 1). The thickness of FLGS flakes was estimated earlier as $2-4 \mathrm{~nm}$ [17]. In addition, we determined this parameter using AFM-microscopy. The best height profile obtained for c-FLGS particles is demonstrated in Figure 2(a). As seen from the figure, the thickness of FLGS flakes is $2-5 \mathrm{~nm}$, which agrees with previous estimation [17]. The thickness of FLGS film was also determined by means of atomic force microscopy. Data obtained for the edge of the film formed by c-FLGS is presented in Figure 2(b). The profile of the edge of a-FLGS film should be the same. Thus, the thickness of FLGS films is $200-300 \mathrm{~nm}$.

According to X-ray photoelectron spectroscopy (Figures $3(\mathrm{a})$ and $3(\mathrm{~d}))$, the content of $\mathrm{O}$ and $\mathrm{S}$ is 18.8 and 1.3 at.\% for c-FLGS and 23.8 and 4.1 at.\% for a-FLGS, respectively. The deconvolution of C1s high resolution XPS spectra (Figures 3 (b) and 3(e)) performed in accordance with $[18,19]$ suggests that oxygen-containing functional groups on the surface of FLGS are represented by epoxy (286.7 eV), hydroxy $(285.3 \mathrm{eV})$, and carboxyl $(288.7 \mathrm{eV})$ groups (Table 1$)$. The deconvolution of the S2p peak (Figures 3(c) and 3(f)) reveals the presence of $\mathrm{C}-\mathrm{SO}_{3}$ group at $169.6 \mathrm{eV}$ for both samples and $\mathrm{C}-\mathrm{SO}_{2}$ at $168.4 \mathrm{eV}$ for c-FLGS [20].

Figure 4 shows current-time curves of FLGS films, obtained by plasma-assisted electrochemical exfoliation of graphite using cathodic (curve 1) and anodic plasma (curve 2). In a dry atmosphere, a current in FLGS film is determined by its electronic conductivity [21]. An increase in the relative humidity always leads to a sharp decrease in the current and a subsequent gradual decrease in its value (Figure 4). The observed decrease in conductivity of FLGS films in a humid atmosphere is associated with the shielding of FLGS flakes by a film of water [21]. A similar decrease in electronic conductivity with an increase in humidity is observed for composite materials [22-24]; this fact is associated with the insulation of electrically conductive particles. Returning 

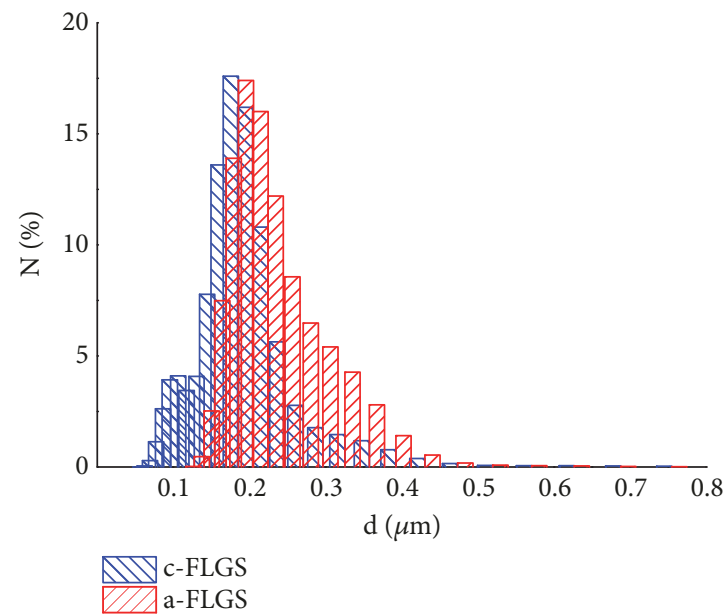

FIGURE 1: Size distribution of c-FLGS (blue) and a-FLGS (red) particles.

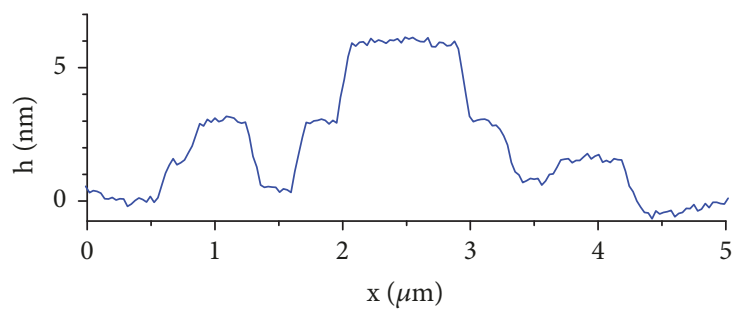

(a)

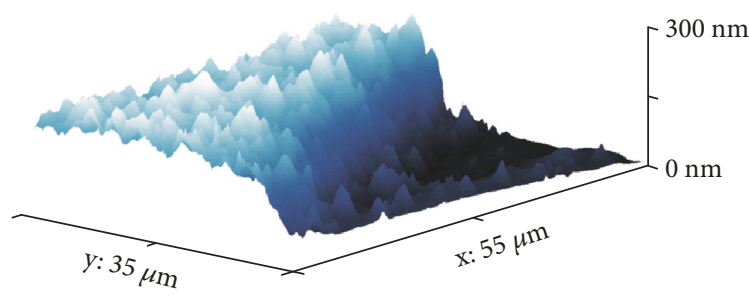

(b)

FIGURE 2: (a) Height profile of c-FLGS particles; (b) AFM image of the edge of c-FLGS film.

a sample to a dry atmosphere leads to an abrupt growth and further slow recovery of the current values. Probably, the reverse process proceeds much slower, since the rate of water molecules removal and restoration of electrical contacts between FLGS flakes is less than the rate of formation of water film around FLGS particles.

Due to the fact that the content of oxygen and sulfur in c-FLGS sample is less than in a-FLGS one, the initial current in a dry atmosphere is somewhat higher for cFLGS film (see curves 1 and 2 in Figure 4). The current drop observed after transition from a dry environment to a humid one is more pronounced for the a-FLGS film. This observation is associated with a high content of carboxyl and hydroxy groups in FLGS produced with anodic plasma. High concentration of hydrophilic groups on the surface of FLGS particles contributes to a more rapid wetting of carbon flakes and diffusion of water molecules into the film. Thus, a slight difference in the nature of change in the values of current for c-FLGS and a-FLGS samples is determined by peculiarities of FLGS composition. In another words, the current in FLGS film is a humidity-dependent parameter defined by concentration of hydrophilic groups on the surface of FLGS (Table 1).

In general, the presence of electronic conductivity of films in a dry atmosphere can be a qualitative criterion for a small degree of functionalization of FLGS surface. However, in our case, the oxygen content in FLGS is sufficiently high and electronic conductivity should be explained by the fact that functional groups are mainly concentrated at the edges of graphene planes, and basal planes themselves do not undergo significant changes compared to the original graphite [20].

With the increase in humidity, two areas can be distinguished on the current-time curve: a rapid decline and a further slow decrease in current due to the diffusion of solvent molecules into FLGS film [25]:

$$
I=A+a e^{-t / \alpha}+b(1-k \sqrt{ } t)
$$

where $A, a, b, \alpha$, and $k$ are parameters of diffusion model, $t$ is the time of solvent molecules diffusion into a film.

Water molecules penetrate into FLGS film with some effective diffusion coefficient $D$ over time $\tau$ [26]. The real diffusion coefficient depends on several factors, such as mass, size, and shape of diffusing molecules, as well as on their chemical nature (polarity). Apparently, the main contribution to the diffusion process is made by the structure (morphology) of FLGS film, which consists of particles with different surface concentration of oxygen-containing functional groups bounded with solvent molecules via hydrogen bonds.

We also observed a change in the conductivity of FLGS films in vapors of solvents of different chemical nature. Figure 5 shows the current-time curves of FLGS in heptane, carbon tetrachloride $\left(\mathrm{CCl}_{4}\right)$, ethanol, and water vapor. The 

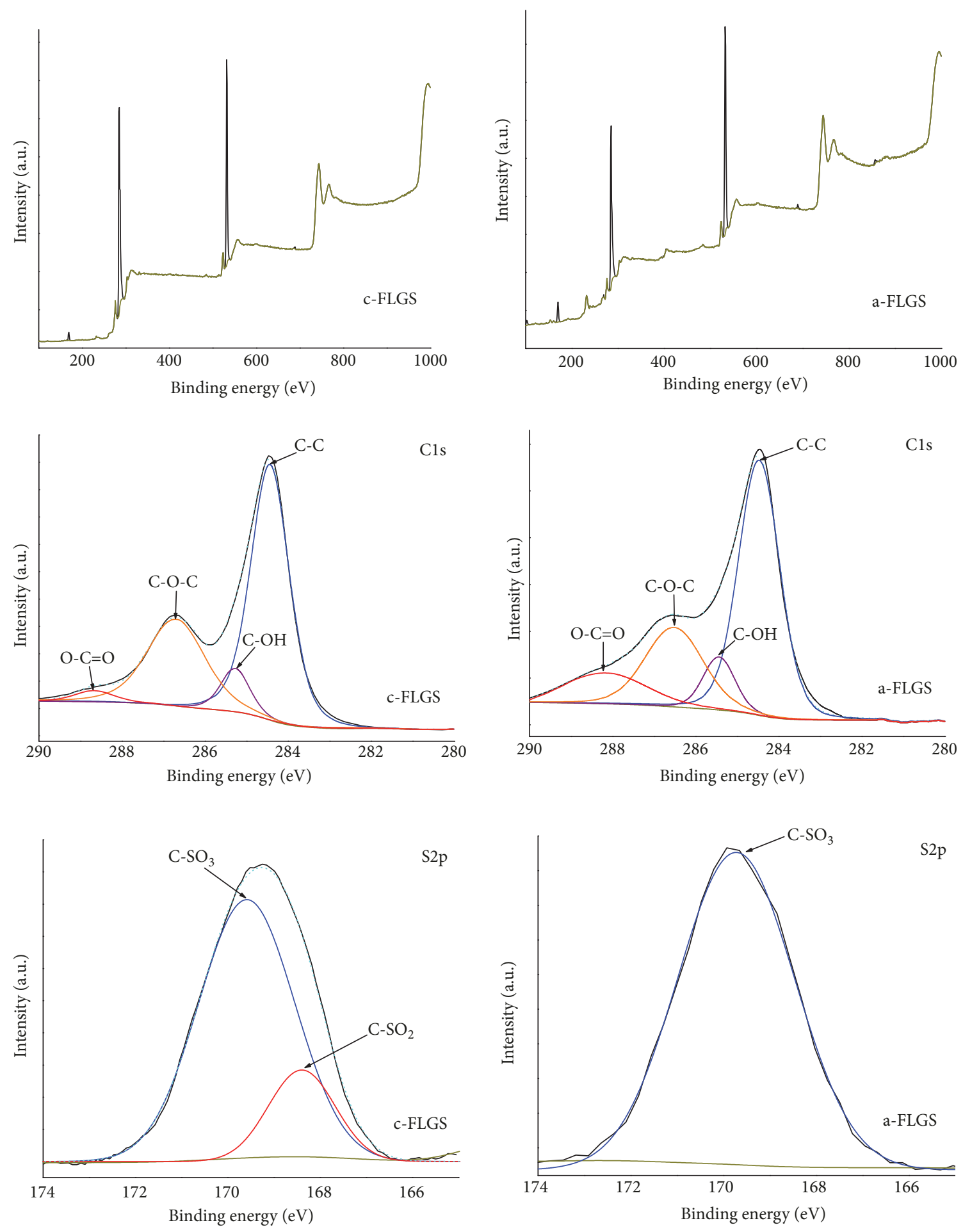

Figure 3: Survey spectra (a, d), high resolution XPS for C1s (b, e), and S2p (c, f) of c-FLGS (a-c) and a-FLGS (d-f).

method of FLGS production (the usage of cathodic or anodic plasma) was not found to distinctly affect the conductivity when the environment in which the sample is located changes.

Despite the high saturation vapor pressure (Table 2 ) in the atmosphere of nonpolar solvents (heptane, $\mathrm{CCl}_{4}$ ), contacts between flakes are not disturbed in FLGS film and it remains conductive (Figure 5). At the same time, in vapors of polar liquids (ethanol, water), there is a significant penetration of solvent molecules into the film and a breakdown of contacts between FLGS flakes, which leads to a decrease in conductivity (the resistance of film increases). Thus, the 


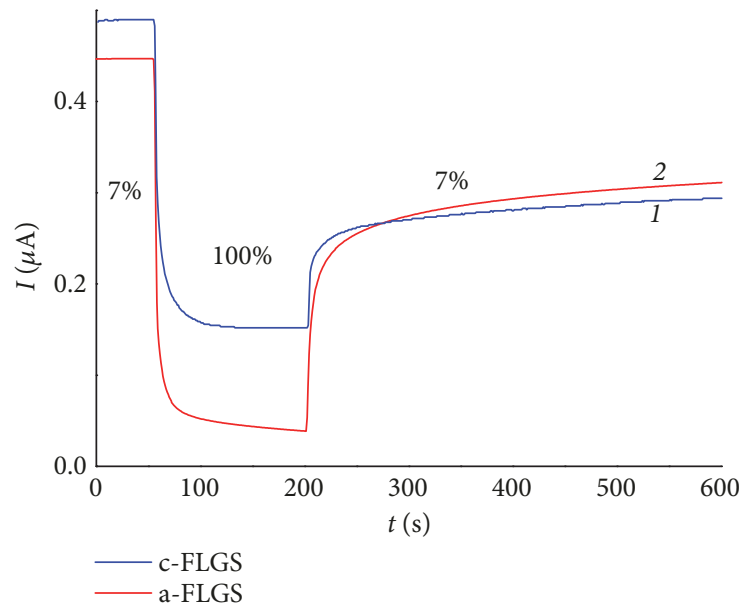

FIgURE 4: The change in the current in FLGS films at different relative humidity of the environment in which the sample is placed.

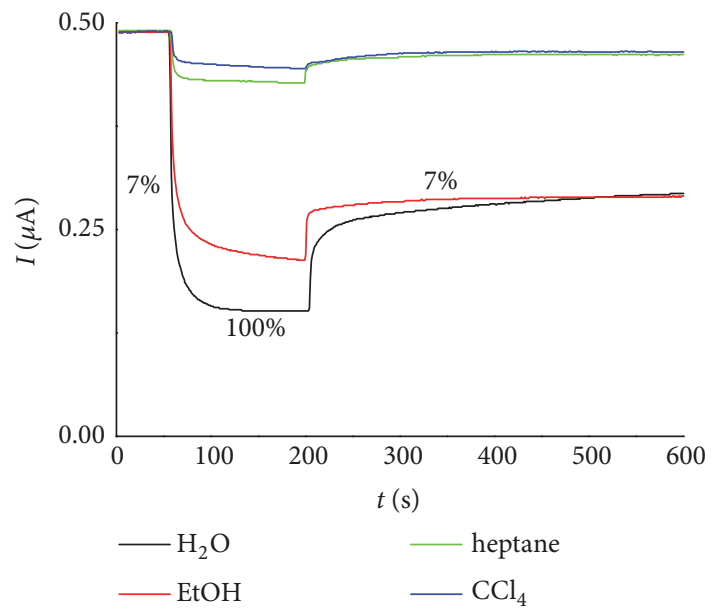

(a)

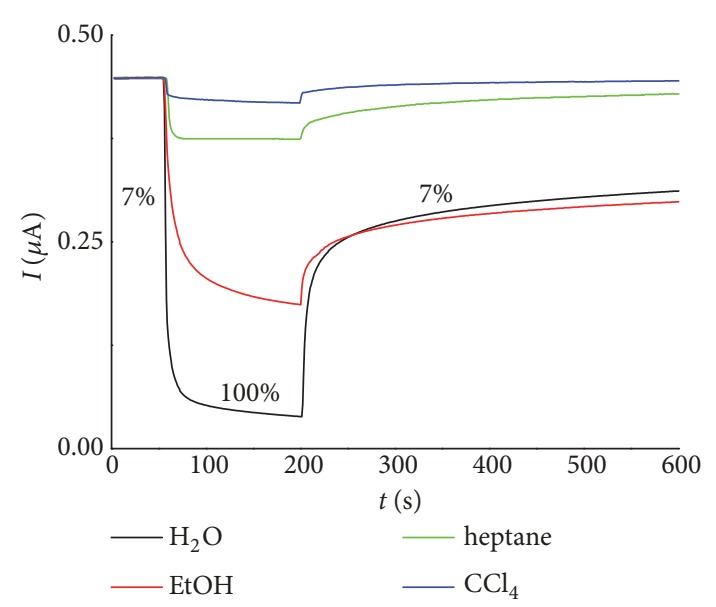

(b)

FIgURE 5: Changes in the conductivity of c-FLGS (a) and a-FLGS films (b) in vapors of solvents of different chemical nature.

TABLE 2: Saturated vapor pressure $P$ and dielectric constant $\varepsilon$ of solvents.

\begin{tabular}{lcc}
\hline Solvent & $P, \mathrm{~mm} \mathrm{Hg}\left(20^{\circ} \mathrm{C}\right)$ & $\varepsilon$ \\
\hline $\mathrm{H}_{2} \mathrm{O}$ & 17.5 & 80.10 \\
EtOH & 43.9 & 24.55 \\
heptane & 35.0 & 1.92 \\
$\mathrm{CCl}_{4}$ & 91.6 & 2.24 \\
\hline
\end{tabular}

presence of polar oxygen-containing functional groups at the edges of graphene planes contributes to the fact that the surface of FLGS flakes becomes hydrophilic, unlike graphene.

\section{Conclusions}

The mode of plasma-assisted electrochemical exfoliation of graphite in $\mathrm{Na}_{2} \mathrm{SO}_{4}$ solution allows one to produce graphene structures with a controlled content of functional groups. It is a relatively cheap and environmentally friendly method of obtaining graphene structures, which can be used in electronic devices, platinum metals free catalysts of oxygen reduction reaction, composite materials, supercapacitors, fuel cells, etc.

In a dry atmosphere, films of few-layer graphene structures possess electronic conductivity without prior reduction (unlike graphene oxide), which indicates the predominant functionalization of edges of graphene planes and the preservation of structure of basal planes in FLGS. The conductivity of FLGS films can be controlled by external conditions, i.e., by increasing or decreasing the humidity of ambient environment. With the increase in humidity, water molecules diffuse into FLGS film. It causes a lessening of the number of contacts between FLGS flakes and, consequently, the current decreases. The influence of chemical nature of solvent on the electrical conductivity of FLGS films is due to the hydrophilic nature of the surface of synthesized FLGS particles. 


\section{Data Availability}

The experimental data used to support the findings of this study are included within the article.

\section{Conflicts of Interest}

The authors declare that they have no conflicts of interest.

\section{Acknowledgments}

The study was financially supported by the Russian Scientific Foundation (project no. 17-73-20236). The work has been performed using the equipment of the Multi-User Analytical Center of IPCP RAS (A. A. Belmesov) and the equipment of the Multi-User Center of Scientific Center in Chernogolovka RAS (E. N. Kabachkov). We are especially grateful to A. I. Lvovsky (Moscow State University of Fine Chemical Technologies) for the particle size distribution analysis.

\section{References}

[1] Y. Wang, X. Chen, Y. Zhong, F. Zhu, and K. P. Loh, "Large area, continuous, few-layered graphene as anodes in organic photovoltaic devices," Applied Physics Letters, vol. 95, no. 6, pp. 063302-063303, 2009.

[2] S. Bae, H. Kim, Y. Lee et al., "Roll-to-roll production of 30-inch graphene films for transparent electrodes," Nature Nanotechnology, vol. 5, no. 8, pp. 574-578, 2010.

[3] D. Wu, F. Zhang, H. Liang, and X. Feng, "Nanocomposites and macroscopic materials: assembly of chemically modified graphene sheets," Chemical Society Reviews, vol. 41, no. 18, p. 6160, 2012.

[4] K. Parvez, S. Yang, Y. Hernandez et al., "Nitrogen-doped graphene and its iron-based composite as efficient electrocatalysts for oxygen reduction reaction," ACS Nano, vol. 6, no. 11, pp. 9541-9550, 2012.

[5] Z. S. Wu, K. Parvez, X. Feng, and K. Müllen, "Graphene-based in-plane micro-supercapacitors with high power and energy densities," Nature Communications, vol. 4, pp. 2487-2488, 2013.

[6] L. Qu, Y. Liu, J.-B. Baek, and L. Dai, "Nitrogen-doped graphene as efficient metal-free electrocatalyst for oxygen reduction in fuel cells," ACS Nano, vol. 4, no. 3, pp. 1321-1326, 2010.

[7] Y. Zheng, Y. Jiao, M. Jaroniec, Y. Jin, and S. Z. Qiao, "Nanostructured metal-free electrochemical catalysts for highly efficient oxygen reduction," Small, vol. 8, no. 23, pp. 3550-3566, 2012.

[8] Q. G. He and E. J. Cairns, "Recent progress in electrocatalysts for oxygen reduction suitable for alkaline anion exchange membrane fuel cells," Journal of The Electrochemical Society, vol. 162, no. 14, pp. 1504-1539, 2015.

[9] X. Liu, L. Li, W. Zhou, Y. Zhou, W. Niu, and S. Chen, "Highperformance electrocatalysts for oxygen reduction based on nitrogen-doped porous carbon from hydrothermal treatment of glucose and dicyandiamide," ChemElectroChem, vol. 2, no. 6, pp. 803-810, 2015.

[10] Y. Fang, H. Wang, H. Yu, and F. Peng, "From chicken feather to nitrogen and sulfur co-doped large surface bio-carbon flocs: an efficient electrocatalyst for oxygen reduction reaction," Electrochimica Acta, vol. 213, pp. 273-282, 2016.
[11] K. Parvez, Z.-S. Wu, R. Li et al., "Exfoliation of graphite into graphene in aqueous solutions of inorganic salts," Journal of the American Chemical Society, vol. 136, no. 16, pp. 6083-6091, 2014.

[12] G. Eda, G. Fanchini, and M. Chhowalla, "Large-area ultrathin films of reduced graphene oxide as a transparent and flexible electronic material," Nature Nanotechnology, vol. 3, no. 5, pp. 270-274, 2008.

[13] S. H. M. Jafri, K. Carva, E. Widenkvist et al., "Conductivity engineering of graphene by defect formation," Journal of Physics D: Applied Physics, vol. 43, no. 4, pp. 045404-045408, 2010.

[14] A. G. Krivenko, R. A. Manzhos, and A. S. Kotkin, "Plasmaassisted electrochemical exfoliation of graphite in the pulsed mode," High Energy Chemistry, vol. 52, no. 3, pp. 272-273, 2018.

[15] A. G. Krivenko, R. A. Manzhos, and A. S. Kotkin, "Pulse generator for electrochemical exfoliation of graphite," Pribory i Tekhnika Eksperimenta, no. 4, pp. 158-159, 2018.

[16] V. Smirnov, V. Vasil'ev, N. Denisov, Y. Baskakova, and V. Dubovitskii, "Electric behavior of interlayer water in graphene oxide films," Chemical Physics Letters, vol. 648, pp. 87-90, 2016.

[17] A. G. Krivenko, R. A. Manzhos, N. S. Komarova, A. S. Kotkin, E. N. Kabachkov, and Y. M. Shul'ga, "Comparative study of graphite and the products of its electrochemical exfoliation," Russian Journal of Electrochemistry, vol. 54, no. 11, pp. 825-834, 2018.

[18] G. Panomsuwan, N. Saito, and T. Ishizaki, "Nitrogen-doped carbon nanoparticle-carbon nanofiber composite as an efficient metal-free cathode catalyst for oxygen reduction reaction," ACS Applied Materials \& Interfaces, vol. 8, no. 11, pp. 6962-6971, 2016.

[19] S. D. Gardner, C. S. Singamsetty, G. L. Booth, G. He, and C. U. Pittman, "Surface characterization of carbon fibers using angleresolved XPS and ISS," Carbon, vol. 33, no. 5, pp. 587-595, 1995.

[20] K. Parvez, R. A. Rincón, N. Weber, K. C. Cha, and S. S. Venkataraman, "One-step electrochemical synthesis of nitrogen and sulfur co-doped, high-quality graphene oxide," Chemical Communications, vol. 52, no. 33, pp. 5714-5717, 2016.

[21] R. A. Manzhos, V. P. Vasilev, and A. G. Krivenko, "Electrical Conductivity of Films Formed by Few-Layer Graphene Structures," Russian Journal of Applied Chemistry, vol. 91, no. 3, pp. 388-391, 2018.

[22] B. A. Aragaw, W. Su, J. Rick, and B. Hwang, "Highly efficient synthesis of reduced graphene oxide-Nafion nanocomposites with strong coupling for enhanced proton and electron conduction," RSC Advances, vol. 3, no. 45, p. 23212, 2013.

[23] M. Tortello, S. Bianco, V. Ijeri, P. Spinelli, and E. Tresso, "Nafion membranes with vertically-aligned CNTs for mixed proton and electron conduction," Journal of Membrane Science, vol. 415-416, pp. 346-352, 2012.

[24] A. Oberoi and J. Andrews, "Metal hydride-nafion composite electrode with dual proton and electron conductivity," International Journal of Smart Grid and Clean Energy, vol. 3, no. 3, pp. 270-274, 2014.

[25] V. Smirnov, N. Denisov, A. Ukshe, and Y. Shulga, "Conductivity of graphene oxide films: Dependence from solvents and photoreduction," Chemical Physics Letters, vol. 583, pp. 155-159, 2013.

[26] E. L. Cussler, Diffusion, Mass Transfer in Fluids Systems, Cambridge University Press, Cambridge, UK, 2nd edition, 1997. 

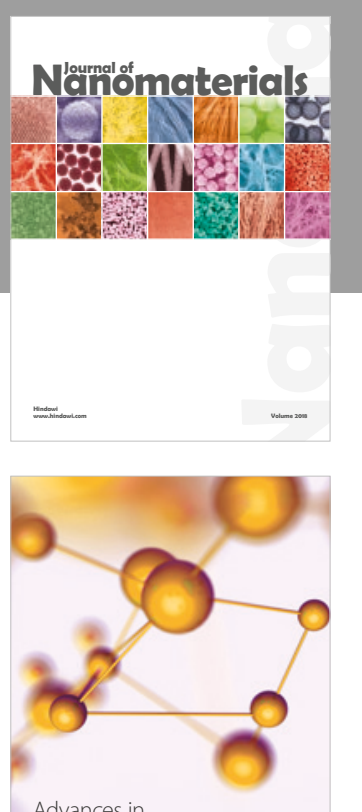

Physical Chemistry
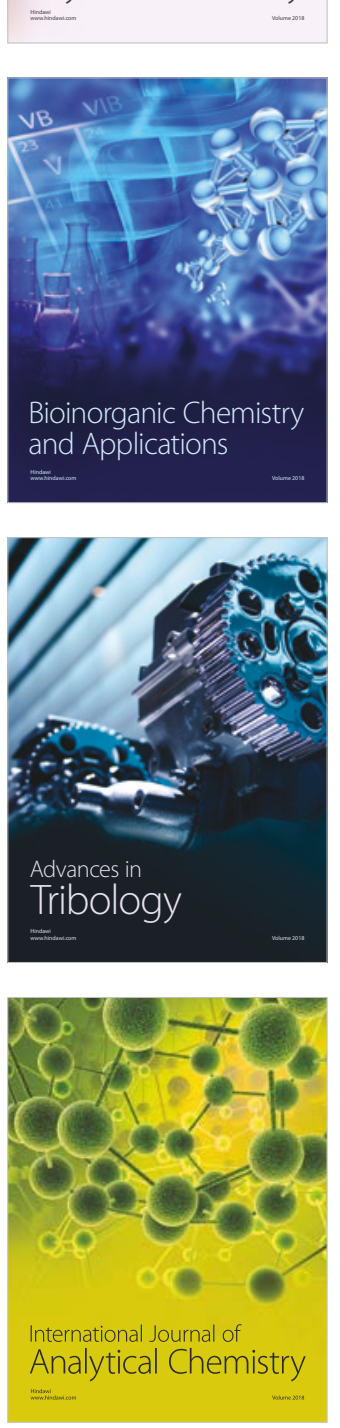

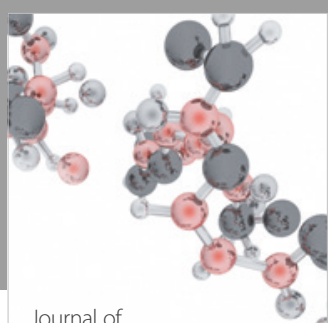

Analytical Methods

in Chemistry

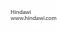

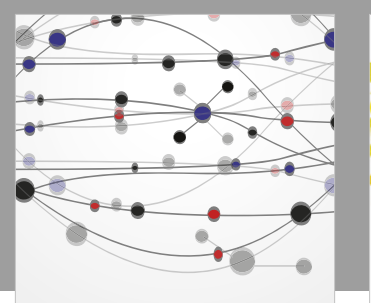

The Scientific World Journal

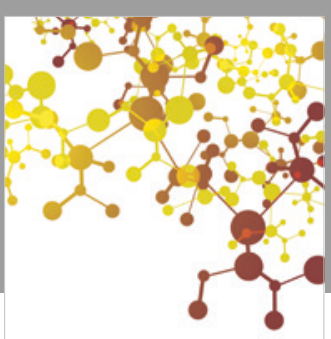

Journal of

Applied Chemistry
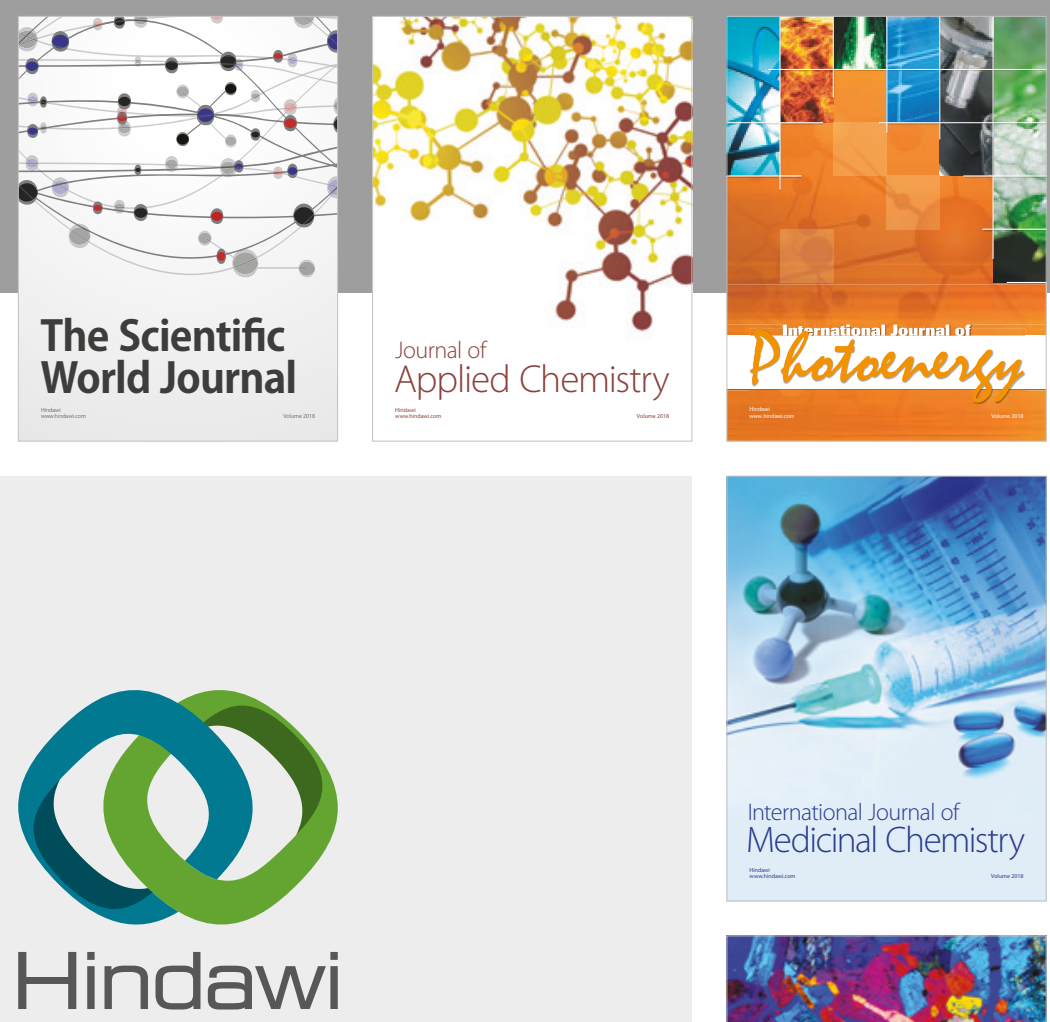

Submit your manuscripts at

www.hindawi.com
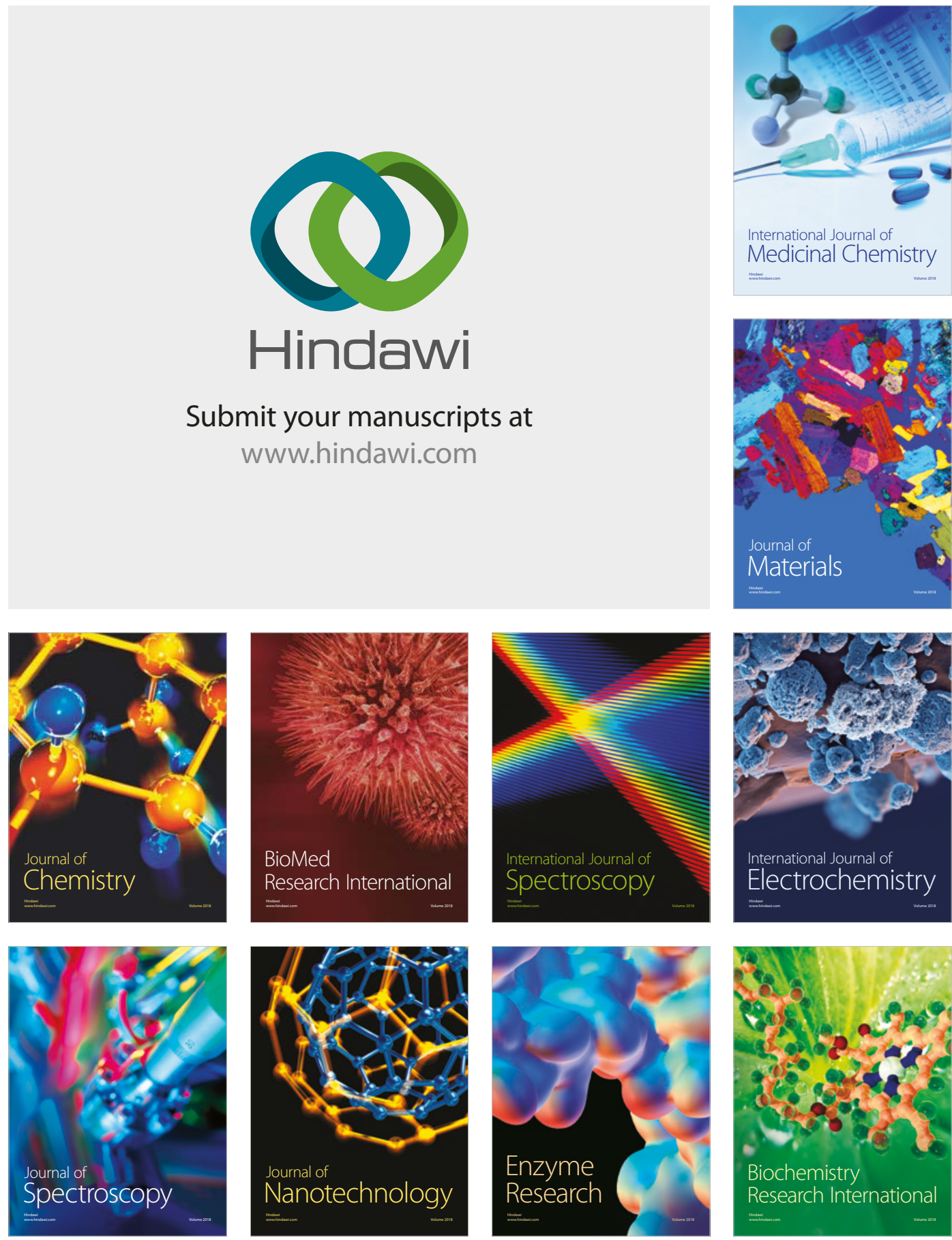
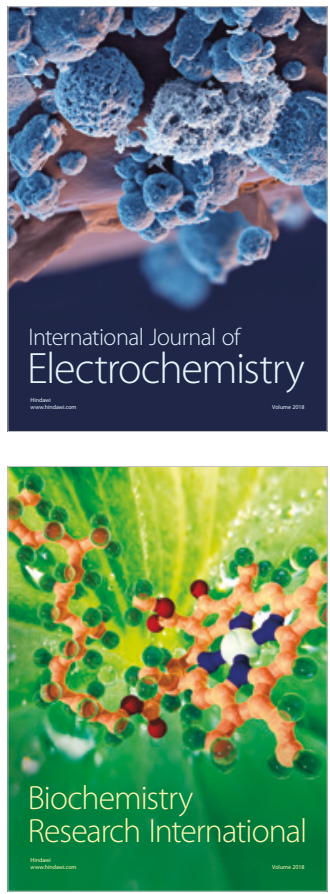